\title{
Quantum backreaction of massive fields and self-consistent semiclassical extreme black holes and acceleration horizons
}

\author{
J. Matyjasek ${ }^{1}$ and O. B. Zaslavskii ${ }^{2}$ \\ ${ }^{1}$ Institute of Physics, Maria Curie-Sklodowska University, \\ pl. Marii Curie-Sklodowskiej 1, 20-031 Lublin, \\ Poland \\ E-mails: matyjase@tytan.umcs.lublin.pl, \\ jurek@iris.umcs.lublin.pl \\ ${ }^{2}$ Department of Physics, Kharkov V.N. Karazin's National University, \\ Svoboda Sq.4, \\ Kharkov 61077, Ukraine \\ E-mail: aptm@kharkov.ua
}

We consider the effect of backreaction of quantized massive fields on the metric of extreme black holes (EBH). We find the analytical approximate expression for the stress-energy tensor for a scalar (with an arbitrary coupling), spinor and vector fields near an event horizon. We show that, independent of a concrete type of $\mathrm{EBH}$, the energy measured by a freely falling observer is finite on the horizon, so that quantum backreaction is consistent with the existence of EBH. For the Reissner-Nordström EBH with a total mass $M_{t o t}$ and charge $Q$ we show that for all cases of physical interest $M_{t o t}<Q$. We also discuss different types of quantum-corrected Bertotti-Robinson spacetimes, find for them exact self-consistent solutions and consider situations in which tiny quantum corrections lead to the qualitative change of the classical geometry and topology. In all cases one should start not from a classical background with further adding quantum corrections but from the quantum-corrected self-consistent geometries from the very beginning.

PACS numbers: 04.70.Dy 


\section{INTRODUCTION}

Nowadays, the physical relevance and importance of the issue of extreme black holes $(\mathrm{EBH})$ does not need detailed clarification. Let us only mention briefly such issues as the end-point of black hole evaporation, information loss, the black hole entropy, etc. In fact, the background of EBH can serve as a promising testing area of potential predictions of (yet not constructed) quantum gravity in the semiclassical domain. Meanwhile, recently, the very fact of existence of semiclassical black holes became the subject of discussion [1]. In this paper Lowe presented strong arguments confirming the existence of semiclassical EBH. These arguments, however, are of phenomenological nature in that they tacitly assume that the components of the stress-energy tensor (SET) of quantized field and their relevant combinations with the metric functions remain finite on the horizon. Meanwhile, this is not obvious in advance. For example, in the background of the Reissner-Nordström (RN) EBH this fact for massless radiation was established only by virtue of thorough numerical calculations [2].

In such a situation it looks reasonable to elaborate the general backreaction approach to EBH similar to that [3] applied to Schwarzschild black holes. However, the attempt of moving in this direction immediately encounters the following difficulty which reveals the crucial difference between the nonextreme and extreme black holes in the given context. In the first case, it was sufficient to choose the fixed background and carry out calculations perturbatively, whereas in the second one the very nature of the background becomes not trivial. Say, for the classical Reissner-Nordström (RN) with charge $Q$ and mass $M$ minor changes around the extreme relationship $M=Q$ can convert EBH to the nonextreme hole or naked singularity. Correspondingly, one should be very careful in examining changes caused by quantum effects at the border of so different kinds of spacetimes. Therefore, the accent in the backreaction program for EBH (at least, on the first step) is to be shifted as compared to the Schwarzschild case: first of all, it is necessary to elucidate, whether or not EBH are compatible with backreaction. It looks natural to take a generic EBH metric, "dressed" 
by surrounding quantum fields, and elucidate, whether or not backreaction is compatible with the property that the Hawking temperature $T_{H}=0$. In turn, this invokes information about the SET of quantized field spacetime of a generic spherically-symmetrical EBH. For massless fields, this task is extremely difficult. Meanwhile, for massive fields recent progress in deriving general expressions for SET [4], [5] makes the task tractable. As the calculation of SET is the key to the problem of existence of quantum-corrected EBH, let us dwell upon this issue in a more detail.

\section{GENERAL FEATURES OF SET OF MASSIVE FIELDS IN CURVED MANIFOLDS}

According to a standard viewpoint, the renormalized stress-energy tensor (SET) of quantized fields evaluated in appropriate state encodes all available information of quantum field theory in curved background, and (beside the classical part) it serves as a source term of the semiclassical Einstein field equations. Unfortunately, mathematical complexities prevent exact analytical treatment and in most of physically interesting situations it cannot be expressed in terms of known special functions. Moreover, what is of principal interest in further applications is not the SET itself evaluated in the particular geometry, but rather its functional dependence on a wide class of metrics. Therefore, we are confronted with two serious problems: construction of the SET on the one hand, and studying the effect of the quantized field upon the spacetime geometry on the other. It is natural therefore, that to address these problems, at least partially, one should employ approximate methods.

It seems that for the massive fields in the large mass limit considered in this paper, an approximation based on the DeWitt-Schwinger expansion is of required generality, allowing, in principle, to attack the problem of backreaction prerturbatively. Moreover, in some situations (also considered here), it is even possible to construct exact solutions of the semiclassical field equations, or, what is more common, guided by physical considerations, guess the appropriate form of the line element. Although such a procedure is limited to espe- 
cially simple geometries with a high degree of symmetries, obtained results are of particular interest and importance.

For the massive fields in a curved spacetime, the renormalized effective action, $W_{R}$, constructed by means of the DeWitt-Schwinger method is given by

$$
W_{R}=\frac{1}{32 \pi^{2} m^{2}} \int d^{4} x g^{1 / 2} \sum_{n=3}^{\infty} \frac{(n-3) !}{\left(m^{2}\right)^{n-2}}\left[a_{n}\right],
$$

where $\left[a_{n}\right]$ is the coincidence limit of the n-th Hadamard-DeWitt coefficient and $m$ is the mass of the field, and the first three terms of the DeWitt-Schwinger expansion have been absorbed by quadratic terms of the generalized classical gravitational action in the process of the renormalization of the bare constants. As the complexity of the Hadamard-DeWitt coefficients rapidly grows with $n$, the practical use of (1) is confined to the first order of $W_{R}$, which involves the integrated coincidence limit of fourth Hadamard-DeWitt coefficient $a_{3}$ computed by Gilkey [6]. Being constructed from local, geometrical quantities, the first order effective action does not describe the process of particle creation which is a nonlocal phenomenon, however, for sufficiently massive fields, the contribution of real particles may be neglected and the DeWitt-Schwinger $W_{R}$ satisfactorily approximates the total effective action. It could be shown that for massive scalar, spinor, and vector fields the first-order effective action could be compactly written in a form [7]

$$
\begin{aligned}
W_{r e n}^{(1)} & =\frac{1}{192 \pi^{2} m^{2}} \int d^{4} x g^{1 / 2}\left(c_{1}^{(s)} R \square R+c_{2}^{(s)} R_{\mu \nu} \square R^{\mu \nu}+c_{3}^{(s)} R^{3}+c_{4}^{(s)} R R_{\mu \nu} R^{\mu \nu}\right. \\
& +c_{5}^{(s)} R R_{\mu \nu \rho \sigma} R^{\mu \nu \rho \sigma}+c_{6}^{(s)} R_{\nu}^{\mu} R_{\rho}^{\nu} R_{\mu}^{\rho}+c_{7}^{(s)} R^{\mu \nu} R_{\rho \sigma} R_{\mu \nu}^{\rho \sigma}+c_{8}^{(s)} R_{\mu \nu} R_{\lambda \rho \sigma}^{\mu} R^{\nu \lambda \rho \sigma} \\
& \left.+c_{9}^{(s)} R_{\rho \sigma}{ }^{\mu \nu} R_{\mu \nu}{ }^{\lambda \gamma} R_{\lambda \gamma}{ }^{\rho \sigma}+c_{10}^{(s)} R_{\mu \nu}^{\rho \sigma}{ }_{\mu} R_{\lambda \gamma}^{\mu \nu}{ }_{\lambda}^{\lambda}{ }_{\rho \sigma}^{\lambda},\right)=\frac{1}{192 \pi^{2} m^{2}} \sum_{i=1}^{10} c_{i}^{(s)} W_{i},
\end{aligned}
$$

where the numerical coefficients depending on the spin of the field are listed in Table I. As the approximate stress-energy tensor is obtained by functional differentiation of (2) with respect to the metric tensor,

$$
T^{\mu \nu(q)}=\frac{1}{96 \pi^{2} m^{2} g^{1 / 2}} \sum_{i=1}^{10} c_{i}^{(s)} \frac{\delta}{\delta g_{\mu \nu}} W_{i},
$$


one concludes that within the framework of the adopted approximation, it is expressed as a linear combination of purely geometrical terms with the numerical coefficients depending on the spin of the field, and consequently is independent of boundary conditions. Since the calculations are carried out for the Euclideanized geometry, the resulting Green functions bear close relations with the temperature Green functions, and in the black hole spacetimes in the absence of the superradiant modes, the thus obtained SET may be interpreted in terms of the thermal state. An alternative approach, consisting in the construction of appropriate Green functions by summing (integrating) WKB approximants of the mode functions of the scalar field equation with arbitrary coupling to a curvature, has been proposed in Ref. 88. It has been shown that, to obtain the lowest order terms in the DeWitt-Schwinger expansion, one has to employ the results of 6-th order WKB. Moreover, detailed analyses, both analytical and numerical, of the stress-energy tensor of the quantized massive scalar field carried out in the Reissner-Nordström spacetime confirmed that the DeWitt-Schwinger approximation yields reasonable results as long as the mass of the field is sufficiently large [8]. Specifically, it was shown that for quantized scalars in the vicinity of the event horizon of RN black hole, the approximation remains within a few percents of the exact (numerical) value if the condition $m M \geq 2$ holds.

General expressions for the first nonvanishing order of SET of the massive scalar, spinor, and vector fields, which generalize earlier results of Frolov and Zel'nikov for vacuum typeD geometries [9], are constructed in [4] and [5]. They may be, in principle, used in any spacetime provided the temporal changes of the background are slow and the ratio of the Compton length to the characteristic radii of curvature are small. However, because of computational complexity, their practical use is limited to simple spacetimes. Happily, there are considerable simplifications for the class of metrics considered in this paper: sphericallysymmetric geometries with vanishing curvature scalar and the spacetimes with maximally symmetric subspaces. On the other hand, however, in some physically important and computationally tractable cases, as for example Kerr or Kerr-Newman spacetimes, there are superradiant modes, and the SET constructed along the lines of the DeWitt-Schwinger ap- 
proximation must be interpreted with care. However, in spite of its inherent limitations, the DeWitt-Schwinger method is still the most general one not restricted to any particular type of symmetry.

In this paper we shall use the general results of [4] and [5] to evaluate the renormalized SETs of the massive scalar, spinor, and vector field in the spacetime of extremal black holes (EBH). The calculations for a general metric turn out to be extremely complicated and are of little practical use. Fortunately, for the issue of the existence of quantum-corrected EBH and the properties of corresponding self-consistent solutions of the Einstein equations, it is sufficient to expand the metric potentials in the vicinity of the event horizon into the Taylor series and examine SET constructed for this simplified line element. Additionally, we shall construct and examine the SET in the Bertotti - Robinson-like spacetimes obtained by expanding the near-horizon geometry into a whole manifold.

\section{SEMICLASSICAL EXTREME BLACK HOLES}

\section{A. quantum backreaction and degenerate horizon}

The metric under consideration reads

$$
d s^{2}=-U d t^{2}+V^{-1} d r^{2}+r^{2} d \Omega^{2}
$$

where the form of $V(r)$ can be found from 00 component of the Einstein equations. It is equal to

$$
\begin{aligned}
V & =1-\frac{r_{+}}{r}-\frac{2 \tilde{m}(r)}{r}, \\
\tilde{m}(r) & =4 \pi \int_{r_{+}}^{r} d r^{\prime} r^{\prime 2} \rho\left(r^{\prime}\right) ;
\end{aligned}
$$

where $\rho=-T_{0}^{0}$, the $\operatorname{SET} T_{\mu}^{\nu}=T_{\mu}^{\nu(c l)}+T_{\mu}^{\nu(q)}$. Here the first term comes from a classical source, the second one is due to the contribution of quantum fields and is to be understood as a quantum average with respect to the the Hartle-Hawking state, renormalized in a proper way. Let us assume that the role of a classical source is played by an electromagnetic field 
$\left(T_{\mu}^{\nu(c l)} \equiv T_{\mu}^{\nu(e m)}\right)$, so we deal with the quantum-corrected RN black hole. Correspondingly, $\tilde{m}(r)=m_{e m}+m_{q}$, where $m_{e m}=\frac{Q^{2}}{2}\left(\frac{1}{r_{+}}-\frac{1}{r}\right), m^{q}=4 \pi \int_{r_{+}}^{r} d r^{\prime} r^{\prime 2} \rho^{q}\left(r^{\prime}\right)$. Here it is implied that the event horizon is located at $r=r_{+}$. In this sense $r_{+}$is "exact" value of the horizon radius (to some extent the word "exact" is conditional since $T_{\mu}^{\nu(q)}$ is known in the one-loop approximation only). The function $U=V e^{2 \psi}$, where the concrete form of the function $\psi(r)$ can be found from the $(r r)$ Einstein equation. Then the Hawking temperature

$$
T_{H}=\frac{V^{\prime}\left(r_{+}\right)}{4 \pi} e^{\psi\left(r_{+}\right)} .
$$

The explicit form of $V$ is

$$
\begin{aligned}
V & =1-\frac{2 m(r)}{r}+\frac{Q^{2}}{r^{2}}, \\
m(r) & =\tilde{m}(r)+\frac{Q^{2}}{2 r}+\frac{r_{+}}{2}=M+m^{q}(r) .
\end{aligned}
$$

It follows from the definitions that $m^{q}\left(r_{+}\right)=0$ (no room for radiation) and $m\left(r_{+}\right)=M$. The condition that $r_{+}$is the root of $V(r)=0$ means that

$$
g(r) \equiv r^{2} V=r^{2}-2 m(r) r+Q^{2}=0 .
$$

If $\psi\left(r_{+}\right)$is bounded on a horizon, the answer to the question whether or not a black hole can reach the extreme state is determined by whether or not $V^{\prime}\left(r_{+}\right)$can turn into zero. In fact, the finiteness of $\psi\left(r_{+}\right)$on the horizon, typical of a nonextreme black hole, becomes a nontrivial issue in the extreme case. It is equivalent to the problem whether or not the energy measured by a freely falling observer remains finite on the horizon (see, cf. [2]). Thorough numerical calculations showed that $\psi\left(r_{+}\right)$is indeed finite for the RN EBH in the case when quantized fields are massless [10]. The behavior of $\psi$ near the horizon is one of key issues examined below in the present paper for the case of massive fields. Let us, however, put for a moment this matter aside and assume that $\psi\left(r_{+}\right)$is indeed finite.

The equation (8) should be satisfied at $r=r_{+}$independently of whether the horizon is extremal. Here $m(r)$ is an unknown function but near $r_{+}$we can expand it like $m(r)=$ 
$M+A\left(r-r_{+}\right)+\ldots$, where $A=4 \pi r_{+}^{2} \rho^{q}\left(r_{+}\right)$has the order $\varepsilon=\hbar / M^{2}$. It is more convenient to write:

$$
m(r)=M_{o}+A r+\ldots
$$

where $M_{0}=M-A r_{+}$. Now we get the equation

$$
g(r)=r^{2}(1-2 A)-2 r M_{0}+Q^{2}=0,
$$

whence the roots are

$$
r_{ \pm}=\frac{M_{0}}{1-2 A} \pm \sqrt{\left(\frac{M_{0}}{1-2 A}\right)^{2}-\frac{Q^{2}}{1-2 A}} .
$$

If one adjust parameters in such a way that $M_{0}^{2}=Q^{2}(1-2 A)$, then

$$
r_{+}=r_{-}=Q / \sqrt{1-2 A}=\frac{M}{1-A}
$$

is the the double root of the function $g(r)$ and $M=\frac{M_{0}(1-A)}{1-2 A}=\frac{Q(1-A)}{\sqrt{1-2 A}}$.

From physical grounds, it is essential for the existence of EBH that the total energy density on the horizon (including, in the RN case, electromagnetic contribution) be positive (and large enough), so the sign of quantum contribution itself (if it is not too large) is not so crucial.

Let us also describe another, seemingly "obvious" approach that, however, contains a hidden trap. In other (less successfull) notations one can write the identity that follows from substitution $r=r_{+}$into $g\left(r_{+}\right)=0$ :

$$
r_{+}^{2}-2 M r_{+}+Q^{2}=0
$$

whence

$$
r_{+}=M+\alpha \sqrt{M^{2}-Q^{2}}
$$

$\alpha=1$ or $\alpha=-1$. At the first glance, the choice $\alpha=1$ should correspond to the event horizon as it is the case for classical RN black holes. However, when one insert $r=r_{+}$ 
into the identity $g\left(r_{+}\right)=0$, it turns out that, as a matter of fact, one inserts into an equation its own root as a parameter of this very equation (by contrast, $M_{0}, Q$ and $A$ are independent parameters and the roots $r_{ \pm}$are expressed in their terms directly according to (11)). This procedure is not quite safe and can lead to the appearance of "spurious" solutions [1]. Therefore, one should verify its self-consistency to make sure that the root under consideration is a "true" one.

It is instructive to demonstrate this explicitly. Let us compare two different presentation of the same quantity - (11) and (14). Then after some manipulations we have

$$
\alpha \sqrt{M^{2}-Q^{2}}=\sqrt{Z}+\frac{M_{0} A}{1-2 A}
$$

where $Z=\left(\frac{M_{0}}{1-2 A}\right)^{2}-\frac{Q^{2}}{1-2 A}$. If $A>0$, one should take $\alpha=1$ (it is supposed that $A$ is not too large; in fact, $A \ll 1)$. However, near the extreme state $Z \rightarrow 0$ and $A<0$, one should choose $\alpha=-1$.

Thus, quantum backreaction shifts the double root to a new position but does not change its character qualitatively [1]. In so doing, however, in a sharp contrast with the classical case, the horizon for $A<0$ lies at $r_{+}=M-\sqrt{M^{2}-Q^{2}}$.

\section{B. General approach to extreme black holes dressed by quantized massive fields}

Meanwhile, as is mentioned above, the fact that backreaction leaves the possibility for the existence of double root of eq. (10) is insufficient in itself for making conclusions about the existence of extreme quantum-corrected black holes. According to $r r$ and $t t$ components of the Einstein equations,

$$
\psi=4 \pi \int_{\infty}^{r} d r F(r), F(r)=r \frac{T_{1}^{1}-T_{0}^{0}}{V} .
$$

Here the lower limit of integration is set to infinity since it is supposed that spacetime infinity is flat and $\psi=0$. Below we will consider the case of massive fields only for which $T_{\mu}^{\nu} \rightarrow 0$ as $r \rightarrow \infty$ (for massless fields it is assumed usually that a system is enclosed into a finite cavity, otherwise $T_{\mu}^{\nu} \rightarrow$ const $\left.\neq 0\right)$. 
The key question is whether or not the quantity $F$ is finite on the horizon. Further details depend on the possibility of power expansion of the metric near the horizon. As far as massless fields are concerned, the counterpart from two-dimensional black hole physics shows [11] that (i) for a generic fixed metric the function $F(r)$ diverges on the horizon that indicates the qualitative change of the metric of extreme black hole under influence of quantum field, (ii) if, instead of fixing a metric in advance, one chooses it as a self-consistent solution of field equations with backreaction taken into account, the existence of an extreme black hole is compatible with quantum backreaction but (iii) the power expansion of the metric near the horizon fails to be analytic. On the other hand, numerical calculations for the four-dimensional RN background [10] showed that $F$ remains finite on the horizon.

There are the following subtleties in our problem. As, by assumption, quantum field is neutral, it does not screen a charge, so its value $Q$ is the same for an original classical and quantum-corrected backgrounds. Then it follows from (11) that $r_{ \pm}<Q$, if $\rho^{q}\left(r_{+}\right)<0$. Had we chosen the classical background with such a relationship between parameters and tried to take into account quantum backreaction by building up the perturbation series, we would have obtained physically meaningless result. Indeed, if the root of the equation $g(r)=0$ is less than $Q$, it corresponds in a classical language to the Cauchy (not the event) horizon, where the stress-energy tensor of quantum fields is known to blow up. This obstacle testifies clearly that, instead of using an standard scheme (pure classical background plus perturbative quantum corrections) we should start from the self-consistent quantumcorrected background from the very beginning.

Our strategy consists in the following. As the issue of the existence of extreme black holes (EBH) demands knowledge of behavior of the metric near the horizon only, let us consider the vicinity of the horizon of a generic $\mathrm{EBH}$, expand the metric near the horizon into the power series and examine, whether or not the quantity $F$ remains finite on the horizon. If $F$ is finite (that means the finiteness of SET in the orthonormal reference frame of a free falling observer [2]), one obtains the power expansion for the SET too, so the full self-consistent solution can be obtained by a direct expansion into the Taylor series with 
respect to $r-r_{+}$.

For EBH the conjectured power expansion in terms of $r-r_{+}$looks like $V=a\left(r-r_{+}\right)^{2}+$ $b\left(r-r_{+}\right)^{3}+\ldots$ For concrete calculations it is more convenient, however, to use, instead of $r$, the proper distance $l$ from some fixed point. Then we have $\frac{d l}{d r}=-\frac{1}{\sqrt{V}}$. Substituting into this equation the power expansion for $V$ near the horizon, we find

$$
r-r_{+}=A_{1} e^{-l / \rho}+A_{2} e^{-2 l / \rho}+\ldots
$$

where $\rho=a^{-1 / 2}$ and the integration constant $l_{0}$ is absorbed by coefficients according to $A_{1}=r_{+} \exp \left(l_{0} / \rho\right), A_{2}=r_{+}^{2} b / a \exp \left(2 l_{0} / \rho\right)$.

We can write down

$$
d s^{2}=-d t^{2} U(l)+d l^{2}+r^{2}(l) d \Omega^{2}
$$

In what follows we assume that power expansion of $U$ in terms of $r-r_{+}$starts from the terms of $\left(r-r_{+}\right)^{2}$, as it is typical for EBH. In term of $l$ this function reads

$$
U=e^{-2 l / \rho} f(l), \quad f=f_{0}+f_{1} e^{-l / \rho}+f_{2} e^{-2 l / \rho} .
$$

The expressions for SET of massive fields in the metric (19) are very cumbersome. However, what is the most important for us, it is their general structure. It turns out that near the horizon

$$
F=F_{0}+F_{1} e^{-l / \rho}+F_{2} e^{-2 l / \rho}+\ldots
$$

with finite coefficients $F_{i}(i=0,1,2 \ldots)$. The expressions for the components of SET read

$$
T_{\mu}^{\nu}=t_{\mu}^{\nu(0)}+t_{\mu}^{\nu(1)} e^{-l / \rho}+t_{\mu}^{\nu(2)} e^{-2 l / \rho}+\ldots
$$

where explicit expression for the coefficients $t_{\mu}^{\nu}$ are listed in Appendix for different kinds of field. It is essential that in all cases it turns out that $t_{0}^{0(0)}=t_{1}^{1(0)}$ and $t_{0}^{0(1)}=t_{1}^{1(1)}$ that just leads to the finiteness of $F$ on the horizon.

It is worth stressing that the finiteness of $F$ for the case of massive fields is shown for any EBH irrespective of whether or not its metric obeys the system of field equations and 
the type of the theory to which these field equation correspond. Now this general result is applied for the most physically interesting case of $\mathrm{RN} E B H$, dressed by its quantum radiation.

\section{C. quantum-corrected RN extreme black hole}

From physical viewpoint, it is natural to fix the total mass measured by a distant observer at infinity (the microcanonical boundary condition). Then we have from (7)

$$
M_{t o t}=M+m^{q}, m^{q}=-4 \pi \int_{r_{+}}^{\infty} d r r^{2} T_{0}^{0(q)}, M=\frac{r_{+}^{2}+Q^{2}}{2 r_{+}} .
$$

The condition of extremality $V^{\prime}\left(r_{+}\right)=0$ entails

$$
r_{+}^{2}(1-2 A)=Q^{2}
$$

In all cases $m^{q}=\alpha_{s} m^{-2} r_{+}^{-3}$, where $\alpha_{0}=-\tilde{\alpha} \frac{17}{441}, \alpha_{1 / 2}=-\tilde{\alpha} \frac{19}{147}, \alpha_{1}=-\tilde{\alpha} \frac{107}{441}$, and $\tilde{\alpha}=\frac{1}{720 \pi}$. From (22), (23) it is seen that the correction of the first order in $M$ cancel and we obtain

$$
M_{t o t}=Q+\alpha_{s} m^{-2} r_{+}^{-3}
$$

In the main approximation $\rho=r_{+}=M=Q$. We see that for all physically relevant cases $\alpha_{s}<0$ and $M_{t o t}<Q$. Thus, a distant observer measuring by precise devices the total mass and charge of an extreme RN black hole, had he relied on classical notions only and neglect quantum backreaction completely, would have been led to the wrong conclusion that in fact the object under investigation is rather naked singularity than a black hole. In other words, the quantum-corrected solution of Einstein-Maxwell equations under discussion not only acquires some small corrections from backreaction of quantum fields but resides in a pure quantum domain where the existence of classical black holes (both extremal or non-extremal) is strictly forbidden.

One can also find the quantum-corrected position of the horizon in terms of physical parameters. Taking into account (12) and noticing that $T_{0}^{0(q)}\left(r_{+}\right)=\eta_{s} m^{-2} r_{+}^{-6}, \eta_{s}=\frac{\mu_{s}}{2880 \pi^{2}}$, $\mu_{0}=\frac{16}{21}-4(\xi-1 / 6), \mu_{1 / 2}=\frac{37}{14}, \mu_{1}=\frac{114}{7}$ 画, we obtain $A=4 \pi \eta_{s} m^{-2} r_{+}^{-4}, r_{+}=Q(1-$ 
$2 A)^{-1 / 2} \approx Q(1+A)$. Equivalently, in terms of a total mass, $r_{+}=M_{\text {tot }}\left(1+\beta_{s} m^{-2} M_{t o t}^{-4}\right)$, $\beta_{s}=4 \pi \eta_{s}-\alpha_{s}$. Here $\beta_{0}=\tilde{\alpha}\left[\frac{353}{441}-4\left(\xi-\frac{1}{6}\right)\right], \beta_{1 / 2}=\tilde{\alpha} \frac{815}{294}, \beta_{1}=\tilde{\alpha} \frac{7289}{441}$.

\section{QUANTUM-CORRECTED BR-LIKE SPACETIMES}

\section{A. Self-consistent solutions without cosmological term}

It is obvious that it is impossible to find exact solution of backreaction equation for realistic four-dimensional $\mathrm{EBH}$ in all space and this is the reason why we were forced to restrict ourselves by the treatment of the vicinity of the horizon only. Meanwhile, there exists another class of objects for which exact solutions (in the one-loop approximation) can indeed be found - metrics with acceleration horizons (Bertotti-Robinson (BR) spacetime and its modifications). Such spacetimes have topology $(r, t) \times S_{2}$, where $S_{2}$ is two-dimensional sphere, so that the coefficient standing at the angular part of a line element is constant. The physical relevance of such spacetimes stems, in particular, from the fact that it can serve as approximation to the true metric of EBH in the vicinity of the horizon. Apart from this, such a kind of a metric appears in the limiting transition from nonextreme black holes to extreme ones [12], [13], [14]. SET for the BR spacetime was studied in [15], [4]. Now, however, we start not from the BR itself, but from its quantum-corrected version.

The general form of metrics under consideration is

$$
d s^{2}=-U(l) d t^{2}+d l^{2}+r_{0}^{2} d \Omega^{2}
$$

where it is assumed that there exists a horizon on which $U \rightarrow 0$. In the coordinates $\left(x^{1}, \theta\right.$, $\phi, t)$ the SET of electromagnetic field is

$$
8 \pi T_{\mu}^{\nu(e m)}=\frac{Q^{2}}{r_{0}^{4}}(-1,1,1,-1) .
$$

However, the expression for SET of quantized fields in such a background is rather complicated and will not be written here. Fortunately, if we restrict ourselves to the BR-like 
spacetime and guess its quantum-corrected version, we obtain a very simple answer in a compact form. We found that this procedure is tractable for the following cases:

Metric $B R 1$ :

$$
\begin{gathered}
d s^{2}=-d t^{2} \rho^{2} s h^{2} \frac{l}{\rho}+d l^{2}+r_{0}^{2} d \Omega^{2}, \\
G_{\mu}^{\nu}=\left(-\frac{1}{r_{0}^{2}}, \frac{1}{\rho^{2}}, \frac{1}{\rho^{2}},-\frac{1}{r_{0}^{2}}\right),
\end{gathered}
$$

Metric $B R 2$ :

$$
d s^{2}=-d t^{2} \exp (-2 l / \rho)+d l^{2}+r_{0}^{2} d \Omega^{2}
$$

The Einstein tensor has the same form (28).

Metric $d S_{2} \times S_{2}$ :

$$
\begin{gathered}
d s^{2}=-d t^{2} \sigma^{2} \sin ^{2} \frac{l}{\sigma}+d l^{2}+r_{0}^{2} d \Omega^{2} \\
G_{\mu}^{\nu}=\left(-\frac{1}{r_{0}^{2}},-\frac{1}{\sigma^{2}},-\frac{1}{\sigma^{2}},-\frac{1}{r_{0}^{2}}\right)
\end{gathered}
$$

Metric Rindler ${ }_{2} \times S_{2}$ :

$$
\begin{gathered}
d s^{2}=-d t^{2} l^{2}+d l^{2}+r_{0}^{2} d \Omega^{2} . \\
G_{\mu}^{\nu}=\left(-\frac{1}{r_{0}^{2}}, 0,0-\frac{1}{r_{0}^{2}}\right)
\end{gathered}
$$

In all cases indicated above SET of quantum fields can be written as

$$
8 \pi T_{\mu}^{\nu(q)}=C\left(f_{1}, f_{2}, f_{2}, f_{1}\right)
$$

where $f_{1}, f_{2}$ are simple constants depending on curvatures of the two dimensional maximally-symmetric subspaces, $C=\frac{1}{12 \pi^{2} m^{2}}$, and $m$ is a mass of a field. The form of (34) follows from the fact that for (27), (29), (30), and (32) the covariant derivatives of the Riemann tensor and its contractions vanish that considerable simplifies the SET given by (3). 
The fact that $T_{2}^{2(q)}=T_{3}^{3(q)}$ is a simple consequence of symmetry of the metric with respect to rotations. The equality $T_{0}^{0(q)}=T_{1}^{1(q)}$ can be understood as follows: the metrics of the type (25) can be obtained as a result of certain limiting transition from black holes ones, in the process of which the near-horizon geometry expands into a whole manifold [14]; then SET pick up their values from the horizon where the regularity condition demands just the validity of this equality.

It turns out that for all cases the function $f_{1}$ and $f_{2}$ share the common general structure. In $B R 1$ and $B R 2 f_{1}=\rho^{-6} r_{0}^{-6}\left(a_{1} r_{0}^{6}+b_{1} r_{0}^{4} \rho^{2}+c_{1} \rho^{6}\right), f_{2}=\rho^{-6} r_{0}^{-6}\left(a_{2} \rho^{6}+b_{2} \rho^{4} r_{0}^{2}+c_{2} r_{0}^{6}\right)$, where the coefficients $a_{i}, b_{i}$ and $c_{i}(i=1,2)$ are the same for both metrics.

For all values of the spin $a_{2}=-a_{1}, b_{2}=-b_{1}, c_{2}=-c_{1}$.

In the scalar case $(s=0)$ :

$$
\begin{aligned}
& a_{1}=\frac{1}{105}\left(8-84 \xi+420 \xi^{2}-840 \xi^{3}\right), b_{1}=-\frac{1}{105}\left(7-112 \xi+630 \xi^{2}-1260 \xi^{3}\right), \\
& c_{1}=\frac{1}{105}\left(4-42 \xi+210 \xi^{2}-420 \xi^{3}\right) ; \\
& s=1 / 2: a_{1}=\frac{20}{420}, b_{1}=\frac{7}{420}, c_{1}=\frac{10}{420} ; \\
& s=1: a_{1}=\frac{8}{35}, b_{1}=\frac{7}{35}, c_{1}=\frac{4}{35} .
\end{aligned}
$$

Metric $d S_{2} \times S_{2}\left(\right.$ now $f_{1}=\sigma^{-6} r_{0}^{-6}\left(a_{1} r_{0}^{6}+b_{1} r_{0}^{4} \sigma^{2}+c_{1} \sigma^{6}\right)$ and $f_{2}=\sigma^{-6} r_{0}^{-6}\left(a_{2} \sigma^{6}+b_{2} \sigma^{4} r_{0}^{2}+\right.$ $\left.\left.c_{2} r_{0}^{6}\right)\right)$ :

$$
\begin{aligned}
& a_{2}=a_{1}, b_{2}=b_{1}, c_{2}=c_{1} . \\
& s=0: a_{1}=\frac{1}{105}\left(-8+84 \xi-420 \xi^{2}+840 \xi^{3}\right), b_{1}=\frac{1}{105}\left(-7+112 \xi-630 \xi^{2}+1260 \xi^{3}\right), \\
& c_{1}=\frac{1}{105}\left(4-42 \xi+210 \xi^{2}-420 \xi^{3}\right) ; \\
& s=1 / 2: a_{1}=-\frac{20}{420}, b_{1}=\frac{7}{420}, c_{1}=\frac{10}{420} ; \\
& s=1: a_{1}=-\frac{8}{35}, b_{1}=\frac{7}{35}, c_{1}=\frac{4}{35} .
\end{aligned}
$$

Metric Rindler $r_{2} \times S_{2}$ :

$$
f_{1}=a r_{0}^{-6}, f_{2}=-2 a r_{0}^{-6}, a_{0}=\frac{8-63 \eta-3780 \eta^{3}}{945}(\eta=\xi-1 / 6), a_{1 / 2}=\frac{1}{42}, a_{1}=\frac{4}{35} .
$$

In the limits $\rho \rightarrow \infty$ and $\sigma \rightarrow \infty$ the metrics BR1and $d S_{2} \times S_{2}$ turn into Rindler ${ }_{2} \times S_{2}$. One can check that in these limits the function $f_{1}$ and $f_{2}$ go smoothly to their values for the metric Rindler ${ }_{2} \times S_{2}$. 
Let the classical background be of the $B R 1$ or $B R 2$ type metrics (The metric (30) cannot appear on the pure classical level without a cosmological constant). Then we obtain two independent equations from the Einstein ones:

$$
\begin{gathered}
-\frac{1}{r_{0}^{2}}=-\frac{Q^{2}}{r_{0}^{4}}+C f_{1} \\
\frac{1}{\rho^{2}}=\frac{Q^{2}}{r_{0}^{4}}+C f_{2}
\end{gathered}
$$

Taking the sum of (35) and (36) and noticing that $f_{1}+f_{2}=\frac{\left(r_{0}^{2}-\rho^{2}\right)}{r_{0}^{6} \rho^{6}} \chi$, where $\chi$ has the structure $\chi=\alpha_{1} r_{0}^{4}+\alpha_{2} \rho^{4}+\alpha_{3} \rho^{2} r_{0}^{2}$ ( $\alpha_{i}$ are pure numbers), we obtain

$$
\left(r_{0}^{2}-\rho^{2}\right)\left(1-\frac{C \chi}{r_{0}^{4} \rho^{4}}\right)=0
$$

Now take into account that $C \sim \lambda_{P L}^{2} \lambda^{2}$, where $\lambda=m^{-1}$ is the Compton length and $\lambda_{P L}$ is the Planckian length. Then a simple estimate shows that the second factor in (37) can turn into zero (provided the proper signs appear in it) for $r_{0} \ll \lambda$ only, so far beyond the region of validity of WKB approximation. Therefore, we will not discuss such a possibility further and assume that there is only one root of eq. (37): $r_{0}=\rho$. This means that BR spacetime remains exact solution of semiclassical equations (cf. [15, 16, 14]). Making use of eq. (35) and writing $f_{1}\left(r_{0}=\rho\right)=\gamma r_{0}^{-6}$, where $\gamma=a_{1}+b_{1}+c_{1}$, we find that $r_{0}^{2}=Q^{2}-C \gamma r_{0}^{-2}$, whence, in the same approximation,

$$
r_{0}^{2}=Q^{2}\left(1-C \gamma Q^{-4}\right)
$$

In (38) the second term in parenthesis represents only a small correction but account for this correction can be crucial in the following sense. Let $\gamma>0$. Then we have $Q>r_{0}$. Let us proceed, for definiteness, in the canonical ensemble approach in which a charge (rather, than a potential on the boundary) should be fixed. First, if $Q \neq r_{0}$, the classical metric with an acceleration horizon of the type (35) or (29) is impossible at all. Instead of it, we would have a geometry of a RN black hole. Second, for $Q>r_{0}$ with $r_{0}$ being the horizon radius, we would have, moreover, a naked singularity. It is clear that the procedure in which 
the ground state is chosen as a classical geometry with a naked singularity with quantum corrections, calculated on such a background perturbatively, is physically unacceptable. Instead of it, we should from the very beginning use the quantum-corrected geometry and check the condition of self-consistency for the corresponding parameters. In our case it is possible due to a relative simplicity of the BR geometry that enables us to find SET just not only for a classical BR itself but also for quantum-corrected version of it (below we will see that it is also the case even if the quantum-corrected metrics changes its form - for example, due to the cosmological term).

The value of $\gamma$ for different values of field spin (subscript indicates the value of a spin $s$ ):

$\gamma_{0}=\frac{5-14 \xi}{105}, \gamma_{1}=\frac{19}{35}, \gamma_{1 / 2}=\frac{37}{420}$. Thus, for a spinor and vector field $\gamma>0$ as well as for the scalar case with the minimal and conformal coupling.

\section{B. Nonzero cosmological constant}

Now for the $B R 1$ and $B R 2$ metrics field equations read

$$
\frac{1}{r_{0}^{2}}=\frac{Q^{2}}{r_{0}^{4}}-\Lambda-C f_{1}
$$

and

$$
\frac{1}{\rho^{2}}=\frac{Q^{2}}{r_{0}^{4}}+\Lambda+C f_{2}
$$

For the $B R 3$ case we have, instead of (40),

$$
\frac{1}{\sigma^{2}}=-\frac{Q^{2}}{r_{0}^{4}}-\Lambda-C f_{2} .
$$

Then it follows from eq. (39) that

$$
\frac{1}{r_{0}^{2}}=\frac{1}{2 Q^{2}} \pm \frac{1}{Q} \sqrt{\frac{1}{4 Q^{2}}+\Lambda+C f_{1}} .
$$

The term with $C f_{1}$ represents a small correction to classical quantities, so that in the expression for $f_{1}$ one can replace $r_{0}$ and $\rho$ by the classical values obtained for $C=0$. If $\Lambda>0$, only the solution with the $+\operatorname{sign}$ should be taken. We will discuss the more 
interesting for us case $\Lambda=-|\Lambda|<0$. Let $\Lambda$ be very close to the value $\Lambda_{0}=-\frac{1}{4} Q^{-2}$ for which the radical in (42) turns into zero. In $f_{1}$ we can put in the main approximation $r_{0}=2^{1 / 2} Q, \rho=\infty$, neglecting corrections of the order $C$. Classically, we would have the product of two-dimensional Rindler and sphere (32), for which, according to the above results, $f_{1}=\bar{a} Q^{-6}, f_{2}=-2 f_{1}$ with $\bar{a}=\frac{1}{8} a>0$ for spinor and vector fields as well as for the scalar case both for the conformal and minimal coupling. If $\Lambda-\Lambda_{0}<0$, the classical constant curvature solutions of the type (35) do not exist at all. However, if the difference $\Lambda-\Lambda_{0}$ is very small and such that $\Lambda-\Lambda_{0}+C \bar{a} Q^{-6}>0$, the solutions (42) do exist. Let us substitute the expression for $r_{0}^{2}$ into eq. (40) for $\rho$. Then

$$
\frac{1}{\rho^{2}}=\frac{1}{r_{0}^{2}}+2 \Lambda+C\left(f_{1}+f_{2}\right)=2\left(\Lambda-\Lambda_{0}\right)+C\left(f_{1}+f_{2}\right) \pm \frac{1}{Q} \sqrt{\Lambda-\Lambda_{0}+C f_{1}} .
$$

Consider two cases.

a) $\Lambda=\Lambda_{0}$. Then we obtain two solutions. The first one is

$$
\rho^{2}=\frac{Q^{4}}{\sqrt{C \bar{a}}}
$$

where the term $-\bar{a} Q^{-6}$ has been dropped. Thus, quantum corrections force the geometry to switch from (32) to (27) or (29). The second solution is formally complex. In fact, this means that instead of (27) we have the geometry (30). Now $\sigma^{2}=\frac{Q^{4}}{\sqrt{C \bar{a}}}$.

2) $\Lambda=\Lambda_{0}-C f_{1}=\Lambda_{0}-C \bar{a} Q^{-6}$. Then we have the metric (30) with parameters

$$
r_{0}^{2}=2 Q^{2}, \sigma^{2}=\frac{1}{C\left(f_{1}-f_{2}\right)}=\frac{Q^{6}}{3 C \bar{a}}
$$

where we took into account the expressions for the metric Rindler $_{2} \times S_{2}$.

\section{CONCLUDING REMARKS}

We have considered EBH in equilibrium with quantized massive fields and demonstrated that for any EBH the components of SET, measured by a free-falling observer, remain finite. If a metric obeys the Eisntein equations, this entails that semiclassical EBH do exist as their self-consistent solutions. The key point of our treatment consisted in restricting to the 
analysis of the near-horizon geometry that enabled us to avoid the complexity connected with obvious impossibility to find explicit self-consistent solutions in the whole domain.

We considered also BR-like spacetime, closely connected to the issue of EBH, and have shown that quantum-corrected BR remain to be the exact solutions of one-loop field equations. In so doing, the relationship between parameters of solutions can be such that classically they are absent at all and only quantum effects make their existence (for fixed values of these parameters) possible. Apart from this, near some critical points in the space of solutions tiny quantum corrections can lead to the change of the type of the BR spacetime, the scale of curvature remaining pure classical. Thus, quantum corrections not only shift slightly the values of relevant physical quantities but lead to qualitative changes in the geometry and topology.

The questions about near-horizon behavior of SET and self-consistent EBH for massless fields as well as properties of self-consistent BR spacetimes deserve separate treatment.

\section{ACKNOWLEDGMENT}

O. Z. thanks Claus Kiefer, Paul Anderson and David Lowe for stimulating discussions and correspondence on quantum-corrected extreme black holes. O. Z. also thanks for hospitality Department of Physics of Freiburg University (where the preliminary stage of this work was started due to kind support of DAAD) and Institute of Physics of Maria Curie-Sklodowska University in Lublin, where the most part of this work has been done.

The authors are grateful to Sergey Sushkov for useful correspondence on BR spacetimes.

\section{APPENDIX A: POWER EXPANSION FOR $\mathbf{T}_{\mu}^{\nu(Q)}$ OF MASSIVE FIELDS NEAR THE HORIZON OF A GENERIC EBH}

In this Appendix we collect a number of formulas for the components of SET of the massive scalar, spinor, and vector fields in the vicinity of the event horizon of a generic eternal black hole, which are used in this paper. Functionally differentiating $W_{i}$ with respect to the 
metric tensor, performing the necessary symmetrizations and simplifications, and inserting thus obtained results into (3) one obtains the general form of the renormalized SET of the quantized massive fields. As the resulting formulas are rather complicated, we shall not display them here, and a reader is referred to [4] and [5] for further details. Subsequently, constructing the components of Riemann tensor, its contractions and necessary covariant derivatives for the line element (18), inserting thus obtained results into the general expressions of SET [5], combining them with the appropriate spin-dependent numerical coefficients $c_{i}^{(s)}$, and finally making use of the explicit form of $U(l)$ and $r(l)$ as given by (19) and (17), and collecting the terms with the like powers of $z=e^{-l / \rho}$, one has

$$
T_{\mu}^{\nu(q)}=\sum_{i=0} t_{\nu}^{\nu(i)} z^{i}=\frac{1}{96 \pi^{2} m^{2}} \sum_{i=0} \widetilde{t}_{\nu}^{\nu(i)} z^{i} .
$$

Although the near-horizon power expansions of the line element (18) look rather simple, the complexity of the SET rapidly increases with the order of expansion, practically invalidating calculations of $t_{\nu}^{\nu(i)}$ for $i \geq 3$. Below the results for $i=0,1$ are listed.

Closer analysis of the coefficients $c_{i}^{(0)}$ given in Table I indicates that the general SET is a third-order polynomial in $\eta=\xi-1 / 6$, with coefficients given by a purely local, geometrical terms.

$$
\begin{aligned}
& \widetilde{t}_{0}^{0(0)}=\widetilde{t}_{1}^{(0)}= \frac{8}{945} \frac{2 r_{+}^{6}+\rho^{6}}{\rho^{6} r_{+}^{6}}-\frac{\eta\left(\rho^{6}-\rho r_{+}^{4}+2 r_{+}^{6}\right)}{15 \rho^{6} r_{+}^{6}}-4 \frac{\eta^{3}\left(2 r_{+}^{6}-3 \rho^{2} r_{+}^{4}+\rho^{6}\right)}{\rho^{6} r_{+}^{6}}, \\
& \widetilde{t}_{0}^{0(1)}= \widetilde{t}_{1}^{(1)}=-\frac{16}{315} \frac{A_{1}\left(r_{+}^{6}+\rho^{6}\right)}{r_{+}^{7} \rho^{6}}+\frac{2}{15} \frac{\eta A_{1}\left(3 \rho^{6}-\rho^{4} r_{+}^{2}-\rho^{2} r_{+}^{4}+3 r_{+}^{6}\right)}{\rho^{6} r_{+}^{7}} \\
&+24 \frac{\eta^{3} A_{1}\left(\rho^{6}+r_{+}^{6}-\rho^{2} r_{+}^{4}-r_{+}^{2} \rho^{4}\right)}{r_{+}^{7} \rho^{6}}, \\
& \widetilde{t}_{2}^{2(0)}=\widetilde{t}_{3}^{3(0)}= \frac{4 \eta^{3}\left(r_{+}^{6}+2 \rho^{6}-3 r_{+}^{2} \rho^{4}\right)}{r_{+}^{6} \rho^{6}}+\frac{\eta\left(\rho^{6}-\rho^{4} r_{+}^{2}+r_{+}^{6}\right)}{15 \rho^{6} r_{+}^{6}}-\frac{2}{945} \frac{\left(8 \rho^{6}+4 r_{+}^{6}\right)}{r_{+}^{6} \rho^{6}} \\
& \widetilde{t}_{2}^{2(1)}=\widetilde{t}_{3}^{3(1)}=\frac{2 A_{1}}{315} \frac{16 \rho^{6}+17 r_{+}^{6}+7 r_{+}^{2} \rho^{4}}{r_{+}^{7} \rho^{6}} \\
&-\frac{\eta}{30 f_{0} \rho^{6} r_{+}^{7}}\left(24 A_{1} f_{0} \rho^{6}+20 A_{1} f_{0} \rho^{4} r_{+}^{2}+3 f_{1} \rho^{4} r_{+}^{3}+40 A_{1} f_{0} \rho^{2} r_{+}^{4}\right.
\end{aligned}
$$




$$
\begin{aligned}
& \left.+100 A_{1} f_{0} r_{+}^{6}+15 f_{1} r_{+}^{7}\right)+\frac{2 \eta^{2}}{f_{0} r_{+}^{5} \rho^{6}}\left(4 A_{1} f_{0} \rho^{4}+3 f_{1} r_{+}^{3} \rho^{2}\right. \\
& \left.+16 A_{1} r_{+}^{4} f_{0}+16 A_{1} r_{+}^{2} f_{0} \rho^{2}+6 f_{1} r_{+}^{5}\right)-\frac{6 \eta^{3}}{f_{0} r_{+}^{7} \rho^{6}}\left(20 A_{1} r_{+}^{2} f_{0} \rho^{4}\right. \\
& +8 \rho^{6} A_{1} f_{0}+8 A_{1} r_{+}^{4} f_{0} \rho^{2}-36 A_{1} r_{+}^{6} f_{0}+3 f_{1} r_{+}^{3} \rho^{4} \\
& \left.+12 f_{1} r_{+}^{5} \rho^{2}-15 f_{1} r_{+}^{7}\right),
\end{aligned}
$$

Repeating the calculations with the coefficients $c_{i}^{(1 / 2)}$ one obtains

$$
\begin{gathered}
\widetilde{t}_{0}^{(0)}=\widetilde{t}_{1}^{1(0)}=\frac{1}{420} \frac{7 r_{+}^{4} \rho^{2}+10 \rho^{6}+20 r_{+}^{6}}{r_{+}^{6} \rho^{6}}, \\
\widetilde{t}_{0}^{(1)}=\widetilde{t}_{1}^{(1)}=-\frac{1}{210} \frac{A_{1}\left(30 \rho^{6}+7 r_{+}^{2} \rho^{4}+7 r_{+}^{4} \rho^{2}+30 r_{+}^{6}\right)}{r_{+}^{7} \rho^{6}}, \\
\widetilde{t}_{2}^{(0)}=\widetilde{t}_{3}^{3(0)}=-\frac{1}{420} \frac{20 \rho^{6}+7 r_{+}^{2} \rho^{4}+10 r_{+}^{6}}{r_{+}^{6} \rho^{6}} \\
\widetilde{t}_{2}^{2(1)}=\widetilde{t}_{3}^{3(1)}=-\frac{1}{840} \frac{21 f_{1} r_{+}^{3} \rho^{4}-136 A_{1} r_{+}^{6} f_{0}-240 \rho^{6} A_{1} f_{0}-140 A_{1} r_{+}^{2} f_{0} \rho^{4}}{f_{0} r_{+}^{7} \rho^{6}}
\end{gathered}
$$

Finally, for the massive vector fields

$$
\begin{gathered}
\widetilde{t}_{0}^{0(0)}=\widetilde{t}_{1}^{(0)}=\frac{1}{35} \frac{8 r_{+}^{6}+7 \rho^{2} r_{+}^{4}+4 \rho^{6}}{\rho^{6} r_{+}^{6}}, \\
\widetilde{t}_{0}^{0(1)}=\widetilde{t}_{1}^{(1)}=-\frac{2}{35} \frac{A_{1}\left(12 r_{+}^{6}+7 \rho^{2} r_{+}^{4}+12 \rho^{6}+7 r_{+}^{2} \rho^{4}\right)}{r_{+}^{7} \rho^{6}}, \\
\widetilde{t}_{2}^{2(0)}=\widetilde{t}_{3}^{3(0)}=-\frac{1}{35} \frac{7 r_{+}^{2} \rho^{4}+8 \rho^{6}+4 r_{+}^{6}}{\rho^{6} r_{+}^{6}}, \\
\widetilde{t}_{2}^{2(1)}=\widetilde{t}_{3}^{3(1)}=-\frac{1}{210 f_{0} r_{+}^{7} \rho^{6}}\left(-280 A_{1} r_{+}^{4} f_{0} \rho^{2}+63 f_{1} r_{+}^{3} \rho^{4}-420 A_{1} r_{+}^{2} f_{0} \rho^{4}\right. \\
\left.-124 A_{1} r_{+}^{6} f_{0}-288 \rho^{6} A_{1} f_{0}+105 f_{1} r_{+}^{5} \rho^{2}\right) .
\end{gathered}
$$

The third-order coefficients of the expansion (A1), $\widetilde{t}_{\mu}^{\nu(2)}$, are too lengthty to be presented here. On the other hand, however, of principal importance in the analyses of the regularity 
of $F$ is the difference between $(t t)$ and $(r r)$ components of the stress-energy tensor rather than the components themselves. The calculations give

$$
T_{t}^{t(q)}-T_{r}^{r(q)}=\frac{1}{96 \pi^{2} m^{2}} \beta z^{2}+\mathcal{O}\left(z^{3}\right)
$$

where

$$
\begin{aligned}
& \beta^{(0)}=-\frac{1}{2520 f_{0}^{2} \rho^{6} r_{+}^{6}}\left(112 A_{1}^{2} f_{0}^{2} \rho^{4}+464 A_{1}^{2} f_{0}^{2} r_{+}^{4}-704 A_{2} f_{0}^{2} r_{+}^{5}\right. \\
& \left.+200 A_{1} f_{0} f_{1} r_{+}^{5}+209 f_{1}^{2} r_{+}^{5}+64 f_{0} f_{2} r_{+}^{6}\right)+\frac{\eta}{30 f_{0}^{2} \rho^{6} r_{+}^{6}}\left(40 A_{1}^{2} f_{0}^{2} \rho^{4}\right. \\
& -8 A_{2} f_{0}^{2} \rho^{4} r_{+}+2 A_{1} f_{0} f_{1} \rho^{4} r_{+}+80 A_{1}^{2} f_{0}^{2} \rho^{2} r_{+}^{2}+168 A_{1}^{2} f_{0}^{2} r_{+}^{4} \\
& +17 f_{1}^{2} \rho^{2} r_{+}^{4}-32 f_{0} f_{2} \rho^{2} r_{+}^{4}-552 A_{2} f_{0}^{2} r_{+}^{5}+42 A_{1} f_{0} f_{1} r_{+}^{5} \\
& \left.+69 f_{1}^{2} r_{+}^{6}-96 f_{0} f_{2} r_{+}^{6}\right)-\frac{\eta^{2}}{2 f_{0}^{2} \rho^{6} r_{+}^{6}}\left(16 A_{1}^{2} f_{0}^{2} \rho^{4}+176 A_{1}^{2} f_{0}^{2} \rho^{2} r_{+}^{2}\right. \\
& -96 A_{2} f_{0}^{2} \rho^{2} r_{+}^{3}+24 A_{1} f_{0} f_{1} \rho^{2} r_{+}^{3}+144 A_{1}^{2} f_{0}^{2} r_{+}^{4}-576 A_{2} f_{0}^{2} r_{+}^{5}-24 A_{1} f_{0} f_{1} r_{+}^{5} \\
& \left.+93 f_{1}^{2} r_{+}^{6}-192 f_{0} f_{2} r_{+}^{6}\right)+\frac{6 \eta^{3}}{f_{0}^{2} \rho^{6} r_{+}^{6}}\left(40 A_{1}^{2} f_{0}^{2} \rho^{4}-8 A_{2} f_{0}^{2} \rho^{4} r_{+}+2 A_{1} f_{0} f_{1} \rho^{4} r_{+}\right. \\
& +64 A_{1}^{2} f_{0}^{2} \rho^{2} r_{+}^{2}-96 A_{2} f_{0}^{2} \rho^{2} r_{+}^{3}+24 A_{1} f_{0} f_{1} \rho^{2} r_{+}^{3}+40 A_{1}^{2} f_{0} r_{+}^{4}+17 f_{1} \rho^{2} r_{+}^{4} \\
& \left.-32 f_{0} f_{2} \rho^{2} r_{+}^{4}+104 A_{2} f_{0}^{2} r_{+}^{5}+46 A_{1} f_{0} f_{1} r_{+}^{5}-8 f_{1}^{2} r_{+}^{6}+32 f_{0} f_{2} r_{+}^{6}\right), \\
& \left.\quad+318 f_{1}^{2} r_{+}^{5}-192 f_{0} f_{2} r_{+}^{5}\right), \\
& \beta^{(1 / 2)}=-\frac{1}{840 f_{0}^{2} \rho^{6} r_{+}^{5}}\left(56 A_{2} f_{0}^{2} \rho^{4}-14 A_{1} f_{0} f_{1} \rho^{4}+288 A_{1}^{2} f_{0}^{2} r_{+}^{3}\right. \\
& -119 f_{1}^{2} \rho^{2} r_{+}^{3}+224 f_{0} f_{2} \rho^{2} r_{+}^{3}+96 A_{2} f_{0}^{2} r_{+}^{4}-96 A_{1} f_{0} f_{1} r_{+}^{4} \\
& +10
\end{aligned}
$$

and

$$
\begin{aligned}
& \beta^{(1)}=-\frac{1}{420 f_{0}^{2} \rho^{6} r_{+}^{5}}\left(336 A_{2} f_{0}^{2} \rho^{4}-84 A_{1} f_{0} f_{1} \rho^{4}+280 A_{1}^{2} f_{0}^{2} \rho^{2} r_{+}+1680 A_{2} f_{0}^{2} \rho^{2} r_{+}^{2}\right. \\
& -420 A_{1} f_{0} f_{1} \rho^{2} r_{+}^{2}+1296 A_{1}^{2} f_{0}^{2} r_{+}^{3}-714 f_{1}^{2} \rho^{2} r_{+}^{3}+1344 f_{0} f_{2} \rho^{2} r_{+}^{3}+1440 A_{2} f_{0}^{2} r_{+}^{4} \\
& \left.-1440 A_{1} f_{0} f_{1} r_{+}^{4}+1935 f_{1}^{2} r_{+}^{5}-2880 f_{0} f_{2} r_{+}^{5}\right),
\end{aligned}
$$

for the quantized massive scalar, neutral spinor, and vector field, respectively. 


\section{APPENDIX B: $\mathrm{T}_{\mu}^{\nu(Q)}$ OF MASSIVE FIELDS IN THE SPACETIME OF EXTREMAL REISSNER-NORDSTRÖM BLACK HOLE}

Although the stress-energy tensor of the massive fields in the spacetime of the extremal Reissner-Nordström black hole may be easily constructed taking extremality limits in the results of Refs. [4] and [8] , below we collect the formulas that have been used in the back-

reaction calculations. For the quantized massive scalar field with an arbitrary curvature coupling one has

$$
\begin{aligned}
T_{0}^{0(q)}= & \frac{M^{2} \eta}{30240 \pi^{2} m^{2} r^{12}}\left(34398 M^{4}-113904 r M^{3}+139944 M^{2} r^{2}-75600 r^{3} M+15120 r^{4}\right) \\
& +\frac{M^{2}}{30240 \pi^{2} m^{2} r^{12}}\left(-1248 M^{4}-45 r^{4}+3084 r M^{3}-2509 M^{2} r^{2}+726 r^{3} M\right), \\
T_{1}^{1(q)}= & -\frac{M^{2} \eta}{30240 \pi^{2} m^{2} r^{12}}\left(4914 M^{4}-21168 r M^{3}+33432 M^{2} r^{2}-23184 r^{3} M+6048 r^{4}\right) \\
& -\frac{M^{2}}{30240 \pi^{2} m^{2} r^{12}}\left(-444 M^{4}-477 r^{4}+1932 r M^{3}-2969 M^{2} r^{2}+1950 r^{3} M\right),
\end{aligned}
$$

and

$$
\begin{aligned}
T_{2}^{2(q)}= & \frac{M^{2} \eta}{30240 \pi^{2} m^{2} r^{12}}\left(44226 M^{4}-143136 r M^{3}+172536 M^{2} r^{2}-91728 r^{3} M+18144 r^{4}\right) \\
& -\frac{M^{2}}{30240 \pi^{2} m^{2} r^{12}}\left(3066 M^{4}-10356 r M^{3}+12953 M^{2} r^{2}-7086 r^{3} M+1431 r^{4}\right),
\end{aligned}
$$

wheras for the massive spinor field one obtains

$$
\begin{gathered}
T_{0}^{0(q)}=\frac{M^{2}}{40320 \pi^{2} m^{2} r^{12}}\left(4917 M^{4}-21496 r M^{3}+32376 r^{2} M^{2}-20080 M r^{3}+4320 r^{4}\right), \\
T_{1}^{1(q)}=\frac{M^{2}}{40320 \pi^{2} m^{2} r^{12}}\left(2253 M^{4}-8680 r M^{3}+12000 r^{2} M^{2}-7120 M r^{3}+1584 r^{4}\right),
\end{gathered}
$$


and

$$
T_{2}^{2(q)}=-\frac{M^{2}}{40320 \pi^{2} m^{2} r^{12}}\left(9933 M^{4}-23552 M r^{3}+42888 r^{2} M^{2}-33984 r M^{3}+4752 r^{4}\right) .
$$

Finally for the massive vector field in the extremality limit one has

$$
\begin{aligned}
& T_{0}^{0(q)}=\frac{M^{2}}{10080 \pi^{2} m^{2} r^{12}}\left(31057 M^{4}-107516 r M^{3}+135391 M^{2} r^{2}-72690 r^{3} M+13815 r\right. \\
& T_{1}^{1(q)}=\frac{M^{2}}{10080 \pi^{2} m^{2} r^{12}}\left(5365 M^{4}-16996 r M^{3}+19349 M^{2} r^{2}-9398 r^{3} M+1737 r^{4}\right),
\end{aligned}
$$

and

$$
T_{2}^{2(q)}=-\frac{M^{2}}{10080 \pi^{2} m^{2} r^{12}}\left(13979 M^{4}+5211 r^{4}-26854 r^{3} M+51789 M^{2} r^{2}-44068 r M^{3}\right) .
$$

TABLE I. The coefficients $c_{i}^{(s)}$ for the massive scalar, spinor, and vector field. Note that to obtain the result for the massive neutral spinor field one has to multiply $W_{\text {ren }}^{(1)}$ by the factor $1 / 2$.

\begin{tabular}{lccc}
\hline \hline & $\mathrm{s}=0$ & $\mathrm{~s}=1 / 2$ & $\mathrm{~s}=1$ \\
$c_{1}^{(s)}$ & $\frac{1}{2} \xi^{2}-\frac{1}{5} \xi+\frac{1}{56}$ & $-\frac{3}{140}$ & $-\frac{27}{280}$ \\
$c_{2}^{(s)}$ & $\frac{1}{140}$ & $\frac{1}{14}$ & $\frac{9}{28}$ \\
$c_{3}^{(s)}$ & $\left(\frac{1}{6}-\xi\right)^{3}$ & $\frac{1}{432}$ & $-\frac{5}{72}$ \\
$c_{4}^{(s)}$ & $-\frac{1}{30}\left(\frac{1}{6}-\xi\right)$ & $-\frac{1}{90}$ & $\frac{31}{60}$ \\
$c_{5}^{(s)}$ & $\frac{1}{30}\left(\frac{1}{6}-\xi\right)$ & $-\frac{7}{720}$ & $-\frac{1}{10}$ \\
$c_{6}^{(s)}$ & $-\frac{8}{945}$ & $-\frac{25}{378}$ & $-\frac{52}{63}$ \\
$c_{7}^{(s)}$ & $\frac{2}{315}$ & $\frac{47}{630}$ & $-\frac{19}{105}$ \\
$c_{8}^{(s)}$ & $\frac{1}{1260}$ & $\frac{19}{630}$ & $\frac{61}{140}$ \\
$c_{9}^{(s)}$ & $\frac{17}{7560}$ & $\frac{29}{3780}$ & $-\frac{67}{2520}$ \\
$c_{10}^{(s)}$ & $-\frac{1}{270}$ & $-\frac{1}{54}$ & $\frac{1}{18}$ \\
\hline \hline
\end{tabular}


[1] D. A. Lowe, Yes, semiclassical zero temperature black holes exist!, gr-qc/0011053.

[2] D.J. Lorantz, W.A. Hiscock, P. R. Anderson, Phys.Rev. D 52, 4554 (1995).

[3] J.W.York, Jr., Phys.Rev. D 31 (1985) 775.

[4] J. Matyjasek, Phys. Rev. D 61, 124019 (2000).

[5] J. Matyjasek, Vacuum polarization of massive scalar fields in the spacetime of an electrically charged nonlinear black hole, gr-qc/0010097, Phys. Rev. D, in press.

[6] P. B. Gilkey, J. Diff. Geom. 10, 601 (1975).

[7] I. G. Avramidi, Nucl. Phys. B355, 712 (1991); Covariant methods for calculation of the effective action in quantum field theory and investigation of higher-derivative quantum gravity, hepth/9510140.

[8] P. R. Anderson, W. A. Hiscock, and D. A. Samuel, Phys. Rev. D 51, 4337 (1995).

[9] V. P. Frolov and A. I. Zel'nikov, Phys. Lett. 115B, 372 (1982); Phys. Lett. 123B, 197 (1983); Phys. Rev. D 29, 1057 (1984).

[10] P. R. Anderson, W. A. Hiscock, and D. J. Loranz, Phys.Rev.Lett. 74, 4365 (1995).

[11] S. Trivedi, Phys. Rev. D 47, 4233 (1993).

[12] O. B. Zaslavskii, Phys. Rev. Lett. 76, 2211 (1996).

[13] O. B. Zaslavskii, Phys. Rev. D 56, 2188 (1997).

[14] O. B. Zaslavskii, Class. Quant. Grav. 17, 497 (2000).

[15] V. Sahni and L. A. Kofman, Phys. Lett. 127B, 197 (2000).

[16] S. N. Solodukhin, Phys. Lett. 448B, 209 (1999). 